\title{
Cotton GhKCH2, a Plant-specific Kinesin, is Low-affinitive and Nucleotide-independent as Binding to Microtubule
}

\author{
Tao Xu, Xuewei Sun, Shiling Jiang, Dongtao Ren and Guoqin Liu* \\ State Key Laboratory of Plant Physiology and Biochemistry, College of Biological Sciences, \\ China Agricultural University, Beijing 100094, China \\ Received 14 February 2007, Accepted 24 April 2007
}

\begin{abstract}
Kinesin is an ATP-driven microtubule motor protein that plays important roles in control of microtubule dynamics, intracellular transport, cell division and signal transduction. The kinesin superfamily is composed of numerous members that are classified into 14 subfamilies. Animal kinesins have been well characterized. In contrast, plant kinesins have not yet to be characterized adequately. Here, a novel plant-specific kinesin gene, $G h K C H 2$, has been cloned from cotton (Gossypium hirsutum) fibers and biochemically identified by prokaryotic expression, affinity purification, ATPase activity assay and microtubulebinding analysis. The putative motor domain of $\mathrm{GhKCH} 2$, $\mathbf{M}_{396-734}$ corresponding to amino acids Q396-N734 was fused with $6 \times$ His-tag, soluble-expressed in $E$. coli and affinity-purified in a large amount. The biochemical analysis demonstrated that the basal ATPase activity of $\mathrm{M}_{396-734}$ is not activated by $\mathrm{Ca}^{2+}$, but stimulated 30-fold max by microtubules. The enzymatic activation is microtubuleconcentration-dependent, and the concentration of microtubules that corresponds to half-maximum activation was about $11 \mu \mathrm{M}$, much higher than that of other kinesins reported. The cosedimentation assay indicated that $\mathbf{M}_{396-734}$ could bind to microtubules in vitro whenever the nucleotide AMP-PNP is present or absent. As a plantspecific microtubule-dependent kinesin with a lower microtubule-affinity and a nucleotide-independent microtubule-binding ability, cotton GhKCH2 might be involved in the function of microtubules during the deposition of cellulose microfibrils in fibers or the formation of cell wall.
\end{abstract}

Keywords: Kinesin, Microtubule motor, ATPase activity, Cosedimentation, Microtubule binding site

\footnotetext{
*To whom correspondence should be addressed.

Tel: 86-10-62733438; Fax: 86-10-62731332

E-mail: Liu@cau.edu.cn
}

\section{Introduction}

Kinesins, as a kind of microtubule-based motors, are involved in controlling many functions associated with microtubules, likely participate in the reorganization of microtubules and the transportation of various materials and organelles within cells (Bloom and Endow, 1994). Kinesins are found universally in all eukaryotic organisms and display varying molecular structures. Conventional animal kinesin heavy chains are composed of (1) a globular motor domain that binds microtubule, hydrolyzing ATP which generates a moving force; (2) an $\alpha$-helical stalk domain that takes part in oligomer formation, and, (3) a tail domain that links light chains or binds various cargoes (Vale and Fletterick, 1997). Almost all kinesins have microtubule-binding ability and microtubulestimulated ATPase activity (Vale and Fletterick, 1997; Matthies et al., 2001). Although motor domains of kinesins are highly conserved, their detail biochemical mechanisms are regarded to be related to the conformational properties of kinesin molecules (Sack et al., 1999; Marx et al., 2005).

Many more kinds of kinesins exist in plant cells, some of which are plant-specific (Matsui et al., 2001; Reddy and Day, 2001; Umeki et al., 2006). For example, AtKCBP in Arabidopsis participates in mitosis and trichome morphogenesis (Reddy and Day, 2000; Vos et al., 2000); AtPAKRP1/2 are associated with phragmoplast organelles (Pan et al., 2004); GhKCH1, a cotton kinesin with $\mathrm{CH}$ (calponin homology) domain, could bind to microfilaments in vitro and occasionally co-localized with transverse-cortical actin microfilaments, and was thought to play a role in organizing the actin network in coordination with the cortical microtubule array in vivo (Preuss et al., 2004). Although a few of plant-specific kinesins are found attaching themselves to specific plant functions including the formation of phragmoplast and cell plate during cell division, morphogenesis of the leaf trichome and so on, both their molecular characters and biochemical properties are known very little.

Plant cells are distinguished from animal cells by the 
presence of a cell wall, chloroplasts and vacuoles. In forming the cell wall, both primary and secondary structures result from the deposition of cellulose microfibrils (Gertel and Green, 1977; Emons and Mulder, 2000). The fibers of cotton are single-cell trichomes differentiated from the epidermis of the ovule. During the development of fibers, there is considerable elongation and thickening of the cell wall. The cellulose deposition has been thought to be related to the microtubule array (Fisher and Cyr, 1998). It is still an open question whether some plant-specific kinesins are involved in the differentiation and development of fibers and whether the directed deposition of cellulose microfibrils is based on the interaction between kinesins and microtubules? Cloning plant-specific microtubule-dependent kinesin genes from cotton fiber and characterizing biochemical properties of their proteins will help us to understand the questions above. In our present study, we cloned a novel kinesin gene $\mathrm{GhKCH} 2$ from cotton fiber, which should be classified into a plant specific group of kinesin-14 subfamily according to its motor domain position and neck-link localization (Lawrence et al., 2004; Richardson et al., 2006). To date, only three members in this kinesin group were reported (Tamura et al., 1999; Preuss et al., 2004; Ni et al., 2005). Our results showed that the prokaryotic-expressed motor domain of GhKCH2 has both microtubule-binding ability and microtubule-stimulated ATPase activity similar to conventional kinesins. However, characteristics are different, especially in its affinity and the manner it binds to microtubules in vitro. These findings provide some useful information for understanding the functions and molecular mechanisms of plant-specific kinesins during the development of cell walls.

\section{Materials and Methods}

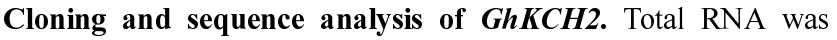
extracted from 15 DPA (day post anthesis) fibers of cotton (Gossypium hirsutum Xuzhou 142) with an RNeasy plant mini kit (QIAGEN GmbH, Hilden, Germany) and used as a template for reverse transcription reaction with M-MLV (Promega) and Oligo $(\mathrm{dT})_{16}$. The degenerated primers corresponding to kinesin's highly conserved motor domain (Mitsui et al., 1993) were used in RT-PCR. The full length of GhKCH2 cDNA was isolated by 5'RACE-PCR and 3'-RACE-PCR according to the manufacturers' instructions (TaKaRa; CLONTECH). Then we obtained the fulllength cDNA including 5'-UTR and 3'-UTR by RT-PCR with LA Taq polymerase (TaKaRa, Dalian, China) using UTR primers (Forward: 5'-CTCTCATTTCAAGCTCCAC-3'; Backward: 5'ACATTTCTCTCTCTCACTCTC-3'). The reported nucleotide acid sequences have been submitted to the GenBank.

Prediction of coiled coils in $\mathrm{GhKCH} 2$ was performed with COILS online at the website: http://www.ch.embnet.org/software/ COILS_form.html. The motif and domain prediction was carried out with SMART (http://smart.embl-heidelberg.de/) and the phylogenetic tree was obtained with DNA STAR MegAlign software by ClustalW method.
Semi-quantitative RT-PCR. Total RNA was extracted from 0, 5 , 10, 15, 20, 25 DPA fibers with the RNeasy plant mini kit. Reverse transcription reaction was performed as described above. The PCR primers, 5'-CATATGGACCAAAGCCAGCACTC-3' (forward) and 5'-GTCGACAGCACTGGCCATATTG-3' (backward) were used for GhKCH2, and 5'-GGTGATGGTGTGTCT-3' (forward) and 5'-ACTGAGCACAATGTTAC-3' (backward) for Actin8.

Prokaryotic-expression and purification of proteins. For expression in and purification from $E$. coli of histidine-tagged GhKCH2 motor domain $\mathrm{M}_{396-734}$ (396-734 aa), a DNA fragment encoding 339 amino acids was amplified by PCR with two oligonucleotide primers (Forward: 5'-GCccatggTTCAGGATCTTA AAGG-3', Backward: 5'-GCaagcttGTTCACTCGAGCAG-3'), and inserted into the NcoI-HindIII site of pET28a (Novagen Inc.). The sequences were verified by sequencing. Fusion protein was expressed in E. coli strain BL21 (DE3) by inducing with $0.2 \mathrm{mM}$ isopropyl- $\beta$-D-thio-galactopyranoside (IPTG) for $8 \mathrm{~h}$ at $22^{\circ} \mathrm{C}$, and purified by affinity chromatography on a column of Ni-NTA agarose (Amersham Pharmacia). The purified fusion proteins were analyzed by SDS-PAGE and identified by western blot using mouse monoclonal anti- $6 \times$ His-tag IgG as the first antibody (R\&D system Inc.) and peroxidase-conjugated goat anti-mouse IgG as the second antibody (Jackson ImmunoResearch Laboratories, Inc.). The membranes were visualized by using an enhanced Lumi-light western blotting substrate kit (Roche).

To prepare microtubules, porcine brain tubulins were purified through three cycles of polymerization and depolymerization according to the published methods (Williams and Lee, 1982; Castoldi and Popov, 2003), and polymerized at $37^{\circ} \mathrm{C}$ for $30 \mathrm{~min}$ in the PEM buffer (100 mM Pipes, pH 6.9, $1 \mathrm{mM}$ EGTA, $2 \mathrm{mM}$ $\mathrm{MgCl}_{2}$ ) plus $1 \mathrm{mM}$ GTP (Sigma) and $10 \mu \mathrm{M}$ taxol (Paclitaxel; a drug that can stabilize microtubules; Sigma).

The protein concentration was determined using the Bio-Rad protein assay kit (Bio-Rad) with BSA (bovine serum albumin) as the standard.

Microtubule-binding assay. Microtubule cosedimentation assays were performed in PEM buffer plus $1 \mathrm{mM}$ DTT and $10 \mu \mathrm{M}$ taxol. $7 \mu \mathrm{M}$ highly purified $\mathrm{M}_{396-734}$ were incubated with $10 \mu \mathrm{M}$ or $0-35$ $\mu \mathrm{M}$ taxol-stabilized microtubules for $10 \mathrm{~min}$ at $25^{\circ} \mathrm{C}$ in the absence or presence of $5 \mathrm{mM} \mathrm{Mg}$-ATP, $5 \mathrm{mM} \mathrm{Na} \mathrm{N}_{2} \mathrm{ATP}, 5 \mathrm{mM}$ ADP or 5 mM 5'-adenylylimidodiphosphate (AMP-PNP; a non-hydrolyzable ATP analogue; Sigma) respectively, then centrifuged at 100,000 g for $30 \mathrm{~min}$ in Beckman ultracentrifuge (Beckman). The supernatants and pellets were separated, brought to equal volumes in the SDS sample buffer, then analyzed by SDS-PAGE and visualized by staining the gel with Coomassie Brilliant Blue R-250. After SDSPAGE analysis, the concentration of $\mathrm{M}_{396-734}$ in the supernatant and pellet was quantified by measuring protein band intensities with Quantity One (Bio-Rad Laboratories, Inc.). Data analysis and affinity constants were made using Microsoft Excel and GraphPad prism $\mathrm{v} 4.03$ software.

ATPase activity assay. Microtubule-stimulated ATPase activity was assayed in PEM buffer except noted. The reaction was started by adding $2 \mathrm{mM}$ ATP in the mixture of $2.5 \mu \mathrm{M}$ taxol-stabilized microtubules and $1 \mu \mathrm{M} \mathrm{M}_{396-734}$, lasted for $15 \mathrm{~min}$ at $25^{\circ} \mathrm{C}$ and 
terminated by adding 10\% TCA (trichloroacetic acid) on ice for $10 \mathrm{~min}$. The supernatant was obtained by a centrifugation at $4^{\circ} \mathrm{C}$, $20,000 \mathrm{~g}$ for $5 \mathrm{~min}$. The inorganic phosphate $(\mathrm{Pi})$ in supernatants was determined as described in published method (LeBel et al., 1978). As a control, TCA was added before the addition of ATP. The assays were repeated three times.

\section{Results}

Isolation of GhKCH2 cDNA. To identify new kinesins expressed in developing cotton fibers, a set of degenerate primers were designed based on the conserved amino acid sequence of kinesin motor domain (Mitsui et al., 1993) and were used in RT-PCR. An approximately $600 \mathrm{bp}$ cDNA fragment was isolated from 15 DPA cotton fibers. After sequencing, a BLAST search of the fragment sequence against GenBank gave a predicted cDNA sequence including a kinesin motor domain. This cDNA fragment is a novel one, shares $89 \%$ amino acid sequence identity with a putative kinesin-related protein (GenBank Accession number: NM_130318) in Arabidopsis (AGI number: At2g47500). Then, 3'- RACE and 5'RACE was carried out to isolate the full length cDNA. After RACE-PCR, we found the polyA in the 3'-UTR and a stop codon in the upstream of 5'-UTR. Running RT-PCR, using the
5'-UTR primer and the 3'-UTR primer, the full-length 3,447 bp cDNA sequence was obtained, and named $\mathrm{GhKCH} 2$ (cotton Kinesin with $\underline{\mathrm{CH}}$ domain 2 , GenBank accession number EF432568) following GhKCH1 identified in cotton by Preuss et al. (Preuss et al., 2004), which contains an open-reading frame of 3,045 bp. The predicted $\mathrm{GhKCH} 2$ protein consisted of 1,015 amino acids with a calculated molecular mass of $112 \mathrm{kDa}$ and a $\mathrm{pI}$ of 7.78 .

Cotton GhKCH2 belongs to kinesin-14 subfamily, and is a new member of plant-specific kinesin with lower identity to conventional kinesin-1. The result of deduced protein sequence analysis indicated that the motor domain of $\mathrm{GhKCH} 2$ is located in the middle region (400-734 aa) of the polypeptide (Fig. 1A) and a unique $\mathrm{CH}$ domain is at the Nterminus of the polypeptide (Fig. 1A). To date, kinesins containing the $\mathrm{CH}$ domain $(\mathrm{KCHs})$ have been found only in plants (Preuss et al., 2004; Richardson et al., 2006).

The kinesin catalytic core has two conserved regions: the residues Iso479 to Thr490 ( ${ }^{479}$ IFAYGQTGSGKT $\left.{ }^{490}\right)$ of GhKCH2 matches the consensus sequence of an ATP binding site (Walker et al., 1990); ${ }^{602} \mathrm{SSRSH}^{606},{ }^{632} \mathrm{DLAGSE}^{637}$ and ${ }^{675} \mathrm{HVPYR}^{679}$ are the characteristic sequences of the microtubule-binding region (Yang et al., 1989).

At the very end of N-terminal side of the motor domain, it
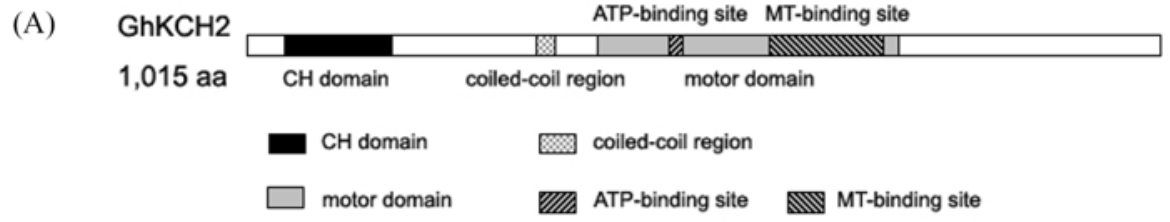

(B)

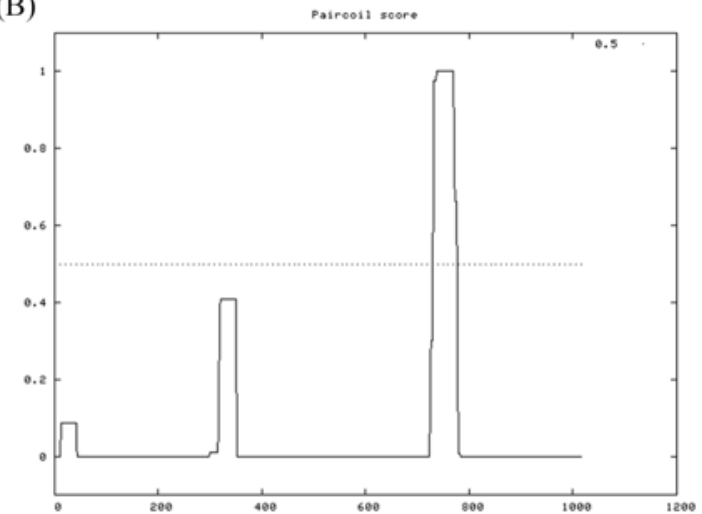

(C)

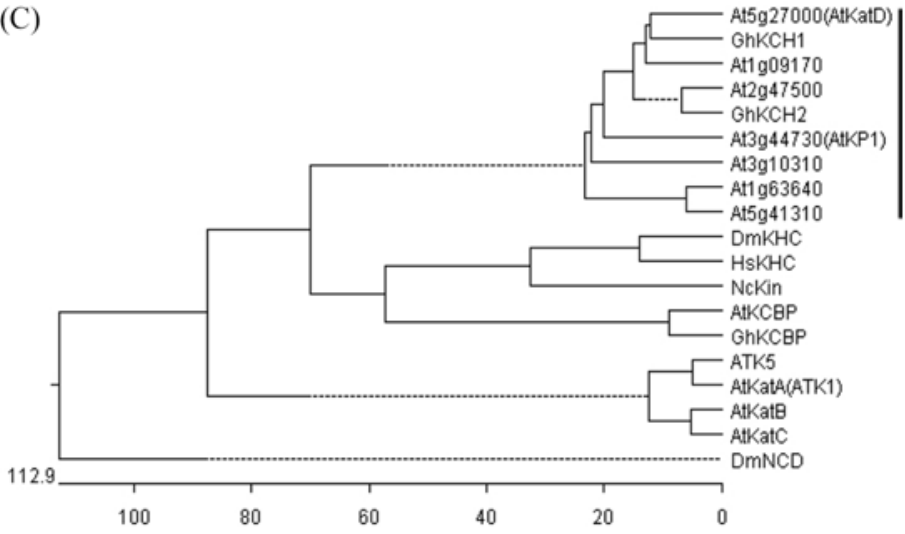

Fig. 1. Sequence analysis of GhKCH2. (A) Schematic diagram for domain organization of GhKCH2 analyzed in SMART (http:// smart.embl-heidelberg.de/), indicating that $\mathrm{GhKCH} 2$ has a putative kinesin motor domain in the central region including conserved ATP-binding site and microtubule (MT)-binding site, a $\mathrm{CH}$ domain at the $\mathrm{N}$-terminal, and a short coiled-coil region at the $\mathrm{N}$-terminal side of motor domain. (B) Prediction of coiled coils by COILS (http://www.ch.embnet.org/software/COILS_form.html). The x axis represents the position of amino acid residues, and the y axis represents the probability of the formation of coiled coils. A probability value greater than 0.5 indicates that the corresponding region most likely forms a coiled-coil. (C) Phylogenetic analysis of GhKCH2 and other representatives of minus end-directed kinesins from plant and fruit fly, and three conventional kinesins from animal and fungal. Phylogenetic analysis of kinesin motor domains was performed with DNA STAR MegAlign software by ClustalW method. The clade containing the KCHs was highlighted by a vertical line. 
contains a sequence of ${ }^{389}$ NRKLYNQVQDLKGS ${ }^{402}$, which matches the consensus neck motif found among kinesins that move toward the minus end of microtubules (Endow, 1999) indicating that $\mathrm{GhKCH} 2$ is probably a microtubule minus end-directed motor. Outside of kinesin motor domain and $\mathrm{CH}$ domain, GhKCH2 showed a large variety in amino acid sequences. The secondary structure prediction revealed two relative short coiled-coil regions (Fig. 1B), one of which is located in the motor domain. In contrast, conventional kinesin has a long coiled-coil region between the motor domain and the tail region. The critical neck interaction residues ${ }^{691} \mathrm{LGG}^{693}$ are present in the catalytic cores (Sack et al., 1997; Case et al., 2000).

The BLAST result indicated that GhKCH2 amino acid sequence had a significantly similarity to the Arabidopsis thaliana putative kinesin-related protein (At2g47500): the motor region shared $88 \%$ and the full-length protein shared $65 \%$ identity. GhKCH2 belongs to the internal kinesin with the $\mathrm{CH}$ domain and shares $57 \%$ identity with cotton kinesin GhKCH1 reported already (Preuss et al., 2004).

To determine the evolutionary relationship between GhKCH2 and other kinesins, a phylogenetic analysis (Fig. 1C) was carried out by examining the motor domain sequence. We found $\mathrm{GhKCH} 2$ and other $\mathrm{KCHs}$ formed a clade. The motor domain of $\mathrm{GhKCH} 2$ shares only $37 \%$ and $42 \%$ identity with that of conventional kinesin or kinesin heavy chain (now named kinesin-1) HsKHC (kinesin heavy chain from Homo sapiens) and NcKin (fungal kinesin from Neurospora crassa).

GhKCH2 motor domain $\left(\mathbf{M}_{396-734}\right)$ was soluble-expressed in $\boldsymbol{E}$. coli and effectively purified to high purity. The above sequence showed a low sequence identity of motor domain between $\mathrm{GhKCH} 2$ and that of conventional kinesin-1. To examine if GhKCH2 motor domain has difference in the common biochemical characteristics (microtubule-binding ability and microtubule-stimulated ATPase activity) from others, a histidine-tagged truncated $\mathrm{GhKCH} 2 \mathrm{M}_{396-734}$ containing the predicted motor domain corresponding to the residues Gln396 to Asn734 was soluble-expressed in E. coli (Fig. 2A, lane 14), purified from the cell extract by Ni-NTA agarose affinity chromatography as a $39 \mathrm{kDa}$ polypeptide (Fig. 2A, lane 5; 2B). Approximately $8 \mathrm{mg}$ of pure $\mathrm{M}_{396-734}$ was prepared from 1 liter of culture by the procedure described above.

The ATPase activity of GhKCH2 motor domain could be activated by microtubules, but with a much higher concentration of microtubules corresponding to the halfmaximum activation. The basal ATPase activity and microtubule-activated ATPase activity of affinity-purified GhKCH2 motor domain $\mathrm{M}_{396-734}$ was measured in PEM buffer. Whereas $\mathrm{M}_{396-734}$ alone showed an ATPase activity of $3.18 \pm 0.49 \mathrm{Pi} \mathrm{mol} \cdot \mathrm{min}^{-1} \cdot \mathrm{mol}^{-1}, 2.5 \mu \mathrm{M}$ microtubules increased the ATPase activity of $\mathrm{M}_{396-734}$ about 7-fold to $22.32 \pm 6.73 \mathrm{Pi}$ $\mathrm{mol} \cdot \mathrm{min}^{-1} \cdot \mathrm{mol}^{-1}$. As a control, microtubules alone showed
(A)

(B)

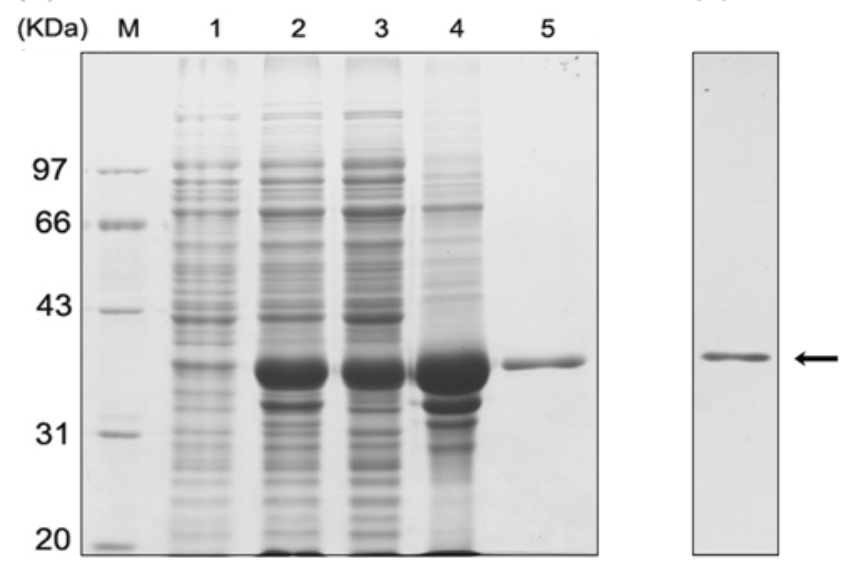

Fig. 2. Prokaryotic expression and affinity purification of Histagged $\mathrm{GhKCH} 2$ motor domain $\mathrm{M}_{396-734}$ fusion proteins. (A) SDS-PAGE analysis of prokaryotic-expressed and affinitypurified $\mathrm{M}_{396-734}$ proteins. Lane $\mathrm{M}$, molecular weight markers. The positions of each band (in kilodaltons) are indicated on the left; Lane 1, total proteins from non-induced E. coli; Lane 2, total proteins from IPTG-induced $E$. coli; Lane 3, soluble proteins from IPTG-induced E. coli; Lane 4, insoluble proteins from induced E. coli; Lane 5, purified soluble $\mathrm{M}_{396-734}$ through Ni-NTA affinity column. (B) Immunoblot of His-tagged $\mathbf{M}_{396-734}$ probed with the monoclonal antibody against $6 \times$ His-tag.

no detectable ATPase activity. Differently, the substitution of $2 \mathrm{mM} \mathrm{Mg}^{2+}$ to $2 \mathrm{mM}$ or $10 \mathrm{mM} \mathrm{Ca}^{2+}$ in the reaction buffer could not dramatically activate the ATPase activity of $\mathrm{M}_{396-734}$, dissimilarly as that of conventional bovine brain kinesin (Kuznetsov and Gelfand, 1986).

Further studies showed that the ATPase activity of $\mathrm{M}_{396-734}$ was activated by microtubules in a concentration-dependent manner (Fig. 3A). The maximum activity was approximately $90 \mathrm{Pi} \mathrm{mol} \cdot \mathrm{min}^{-1} \cdot \mathrm{mol}^{-1}$, about 30 -fold activated. And the microtubule concentration at half-maximum activation $\left(K_{m}\right)$ was $11.15 \pm 0.83 \mu \mathrm{M}$ in the low ionic strength buffer PEM. More interestingly, in the absence of microtubules, the basal ATPase activity of $\mathrm{M}_{396-734}$ was not affected obviously by different $\mathrm{NaCl}$ concentration; but in the presence of microtubules, its ATPase activity is decreased as the concentration of $\mathrm{NaCl}$ increased, indicating that the degree of microtubule activation of $\mathrm{M}_{396-734}$ was sensitive to the ionic concentration (Fig. 3B). As to $\mathrm{pH}$ dependency, the maximum microtubule-activated ATPase activity was observed at $\mathrm{pH}$ 6.5 , but in the absence of microtubules, value of the ATPase activity showed no peak as the buffer $\mathrm{pH}$ changed (Fig. 3C).

The binding of GhKCH2 motor domain to microtubules is nucleotide-independent. Most Kinesins can bind specifically to microtubules in the presence of the nucleotide AMP-PNP. In order to know if $\mathrm{GhKCH} 2$ motor domain $\mathrm{M}_{396-734}$ has microtubule-binding ability, the purified $\mathrm{M}_{396-734}$ was incubated with taxol-stabilized microtubules in the absence or presence 
(A) [MT] dependence of the ATP ase activity

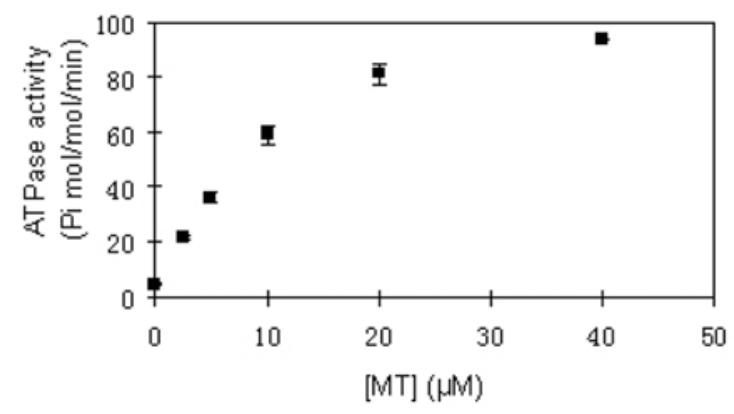

(B) $[\mathrm{NaCl}]$ dependence of the ATPase activity

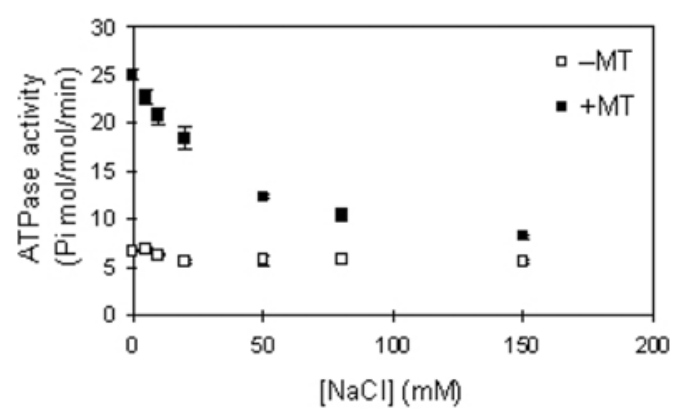

(C) $\mathrm{pH}$ dependence of the ATPase activity

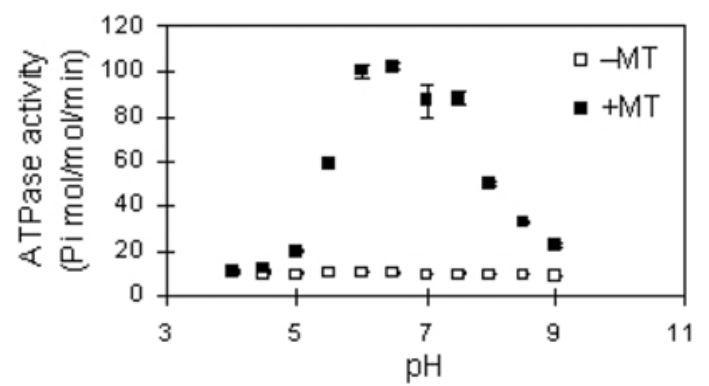

Fig. 3. ATPase activity assays of the GhKCH2 motor domain $M_{396-734}$. (A) Microtubule concentration dependence of $M_{396-734}$ ATPase activity. $1 \mu \mathrm{M} \mathrm{M}_{396-734}$ was incubated with $0-40 \mu \mathrm{M}$ microtubules for $15 \mathrm{~min}$ at $25^{\circ} \mathrm{C}$ in PEM buffer containing $2 \mathrm{mM}$ ATP. (B) ATPase activity of $\mathrm{M}_{396-734}$ in different concentrations of $\mathrm{NaCl}$. Reactions were performed by adding $2 \mathrm{mM}$ ATP in $1 \mu \mathrm{M} \mathrm{M}_{396-734}$ in PEM buffer containing $0-150 \mathrm{mM} \mathrm{NaCl}$, and incubating for $15 \mathrm{~min}$ at $25^{\circ} \mathrm{C}$, in the absence (open squares) or presence (closed squares) of $2.5 \mu \mathrm{M}$ microtubules. (C) $\mathrm{pH}$ dependence of the ATPase activity of $\mathrm{M}_{396-734}$. ATPase assays were carried out at $25^{\circ} \mathrm{C}$ in buffers with $2 \mathrm{mM}$ $\mathrm{MgCl}_{2}, 1 \mathrm{mM}$ EGTA and $1 \mu \mathrm{M} \mathrm{M}_{396-734}$ in the absence (open squares) or presence (closed squares) of $2.5 \mu \mathrm{M}$ microtubules. The ATPase reaction was started by adding $2 \mathrm{mM}$ ATP. The buffers used are: acetate- $\mathrm{NaOH}$ for $\mathrm{pH}$ 4.0-5.0, MES-NaOH for $\mathrm{pH} 5.5-6.5$, MOPS$\mathrm{NaOH}$ for $\mathrm{pH}$ 7.0-7.5, and Tris- $\mathrm{HCl}$ for $\mathrm{pH} 8.0-9.0$.

of AMP-PNP, ATP and ADP, and presumptive microtubule$\mathrm{M}_{396-734}$ complex was then isolated by centrifugation (Fig. 4A). The level of $\mathrm{M}_{396-734}$ cosedimentated with microtubules in the presence of AMP-PNP was much higher than that in the presence of ATP or ADP, consist with that of most other kinesins. Notably, even in the absence of AMP-PNP, M $_{396-734}$ also could bind to microtubules, indicating that $\mathrm{GhKCH} 2$ has a nucleotide-independent microtubule-binding ability.

The microtubule-binding of $\mathrm{M}_{396-734}$ was measured in the absence or presence of ADP and ATP for a range of microtubule concentrations. Binding curves are shown in Fig. 4B. The maximum microtubule-binding ratio of $\mathrm{M}_{396-734}$ (Fig. $4 \mathrm{~B})$ in the absence of nucleotides was 0.42 , and the microtubule-dissociation constant $\mathrm{K}_{d}(\mathrm{MT})$, is $7.69 \mu \mathrm{M}$. The $\mathrm{K}_{\mathrm{d}}(\mathrm{MT})$ of $\mathrm{M}_{396-734}$ increased to $14.65 \mu \mathrm{M}$ in the presence of ATP, little higher than $12.98 \mu \mathrm{M}$ in the presence of ADP.

\section{Discussion}

The aim of the present study was to characterize a plant specific kinesin $\mathrm{GhKCH} 2$ cloned from cotton fiber. We demonstrated that although the protein sequence of $\mathrm{GhKCH} 2$ motor domain is only $30-40 \%$ identity with that of conventional kinesin- 1 , it still keeps the distinct biochemical properties of kinesins. The soluble prokaryotic-expressed $\mathrm{GhKCH} 2$ motor domain $\mathrm{M}_{396-734}$ has ATPase activity that could be stimulated by microtubules, and could bind to microtubules in vitro (Fig. 3A, 4A). Our results demonstrate that cotton $\mathrm{GhKCH} 2$ is a microtubuledependent kinesin motor gene.

The ATPase activities of the prokaryotic-expressed plant kinesin motor domain have rarely been reported (Mitsui et al., 1994; Tamura et al., 1999; Umeki et al., 2006). Detail biochemical analysis of the GhKCH2 motor domain $\mathrm{M}_{396-734}$ indicated that either ATPase activity or microtubule-binding ability of GhKCH2 exhibits several specific properties: (1) the basal ATPase activity of $\mathrm{M}_{396-734}$ could only be stimulated by the addition of microtubules, but not activated by $\mathrm{Ca}^{2+} ;(2)$ the ATPase activity of $\mathrm{M}_{396-734}$ was activated by microtubules in a concentration-dependent manner (Fig. 3A). The magnitude of the enhancement was tens of folds, similar as that of other prekaryotic-expressed plant kinesin motors (Tamura et al., 1999; Umeki et al., 2006), but much lower than that for many animal kinesins (Kuznetsov and Gelfand, 1986; Matthies et 

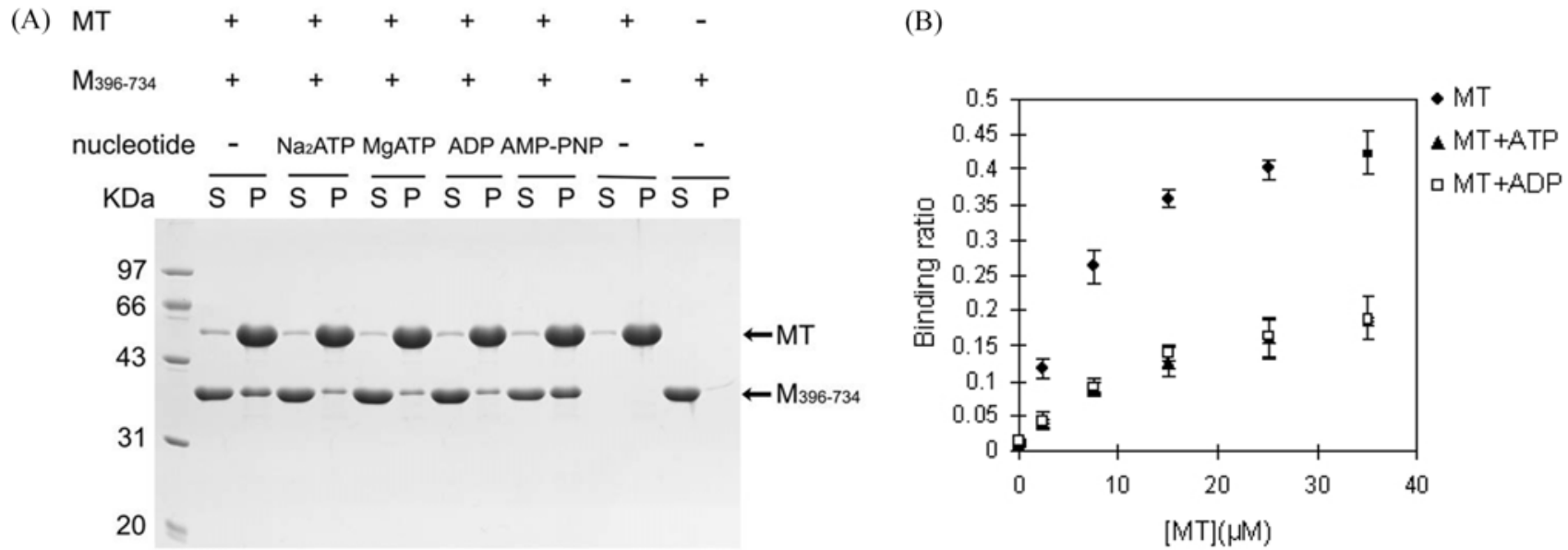

Fig. 4. Microtubule cosedimentation of GhKCH2 motor domain $\mathrm{M}_{396-734}$. (A) $7 \mu \mathrm{M} \mathrm{M}$ 396-734 was used for microtubule-binding in the absence or presence of $5 \mathrm{mM} \mathrm{Na} 2 \mathrm{ATP}$, Mg-ATP, ADP or AMP-PNP. Resulting proteins in the supernatant (S) and pellet (P) are shown. (B) Binding curves of $\mathrm{M}_{396-734}$ to microtubules $(0-35 \mu \mathrm{M})$ in the absence of AMP-PNP, and in the presence of ATP or ADP. Microtubule binding ratio $=$ the concentration of $\mathrm{M}_{396-734}$ bound to microtubules/total concentration of $\mathrm{M}_{396-734}$.

al., 2001); (3) the microtubule concentration for half-maximum activation of $\mathrm{GhKCH} 2\left(K_{m}\right)$ was about $11 \mu \mathrm{M}$ in the low ionic strength buffer PEM (Fig. 3A), approximately 20-fold higher than those of kinesin-1 (Ma and Taylor, 1995; Klumpp et al., 2003). This is a little higher than that of rice kinesin K16 (Umeki et al., 2006); (4) Nucleotide AMP-PNP could enhance the binding of GHKCH2 to microtubules, but in the absence of AMP-PNP, GhKCH2 can also bind to microtubules more readily than in the presence of ATP and ADP-about a 2-fold increase (Fig. 4A, Fig. 4B), indicating that the interaction of GhKCH2 with microtubules was partially through its enzyme activity center, and partially through its other sites which are not related to its ATPase activity.

The stable basal ATPase activity, the less microtubuleactivated ATPase activity, the higher $K_{m}$ (MT), and the nucleotide-independent microtubule-binding manner, are likely to be due to a different 3-D molecular structure of $\mathrm{GhKCH} 2$, and should reflect different functions in plant cells. We predicated 3D-model of GhKCH2 motor domain $\mathrm{M}_{396-734}$ using BioInfoBank MetaServer, and compared it with that of a kinesin-1 member from fungal Neurospora crassa, NcKin (PDB-ID is 1GOJ) which is regarded as the best model due to the highest Jscore (209.00), based on Delano Scientific LLC PyMOL software (Fig. 5A). There are two obvious differences between the two models: one lies in loop 8 (L8) that in $\mathbf{M}_{396-734}$ (Fig. 5A-b, c, in purple) extrudes much from the surface of the molecule, and another lies in Loop 12 (L12) that in $\mathbf{M}_{396-734}$ (Fig. 5A-a, b, in purple) appeared as a relaxed loop. It is believed that the structural elements of kinesins that contribute most to the interaction with microtubules are (1) the $\beta$-sheet 5 to the L8, this is the $\beta 5$-L8 lobe, (2) the switch II region composed of two loops and two $\alpha$-helixes, this is L11- $\alpha 4$ L12- $\alpha 5$ cluster (Sack et al., 1999; Song et al., 2001; Marx et al., 2005). Primary structure comparisons with NcKin indicated that the $\mathrm{L} 8$ of $\mathrm{GhKCH} 2$ contains more residues with hydroxyl groups and charged $\mathrm{R}$ groups (T, S, K, R, E), and fewer amide residues and Pro, but the L12 in Switch II of $\mathrm{GhKCH} 2$ contains fewer residues with hydroxyl groups and charged $\mathrm{R}$ groups and more amide residues and Pro (Fig. 5B). The divergent conformation of L8, L12 in GhKCH2 motor domain may account for the weaker interaction between $\mathbf{M}_{396-734}$ and microtubules.

The dissociation constant of GhKCH2 motor domain from microtubules in the presence of ATP is slightly larger than that in the presence of ADP, but the difference is small. This result is consistent with the NCD motor protein (Shimizu et al., 1995). ATP hydrolysis is based on a cycle of conformational changes. The Kinesins must be firstly attached to their microtubule track, ATP is then hydrolyzed, phosphate is released, and the kinesins are left in the ADP-bound state with a greatly reduced affinity for microtubules (Matthies et al., 2001). Kinesin has been shown to contain tightly bound ADP even after purification (Hackney, 1988; Shimizu et al., 1995).

We do not know the specific functions of cotton GhKCH2. However, from our results of the semi-quantitative RT-PCR analysis (Fig. 6), GhKCH2 may be related to the functions of microtubule during the formation of cell wall of fibers. GhKCH2 gene was expressed in all the stages of fiber development: from 0 to $10 \mathrm{DPA}$, expression level increased, from 10 to 15 DPA up to the max level, and from 20-25 DPA expression levels decreased. It is very similar to the expression pattern of tubulin in cotton fibers (Kloth, 1989). During the fiber elongation period (5-25 DPA), cortical microtubules align in a parallel manner, perpendicular to the axis of elongation. Fiber elongation completes and cell wall deposition begins between 10 and 20 DPA. At the onset of secondary cell wall synthesis, parallel microtubules align in a steeply pitched manner (Seagull, 1992). The cortical microtubules array 
(A)

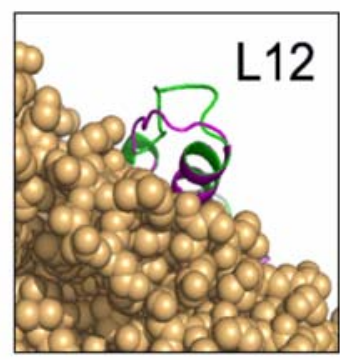

a

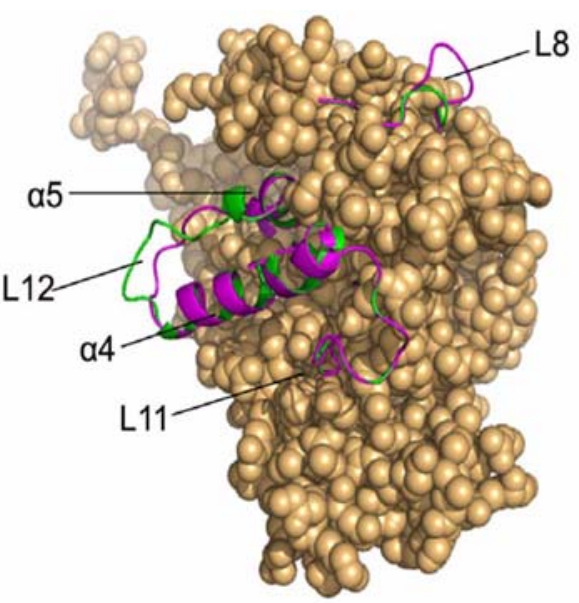

b

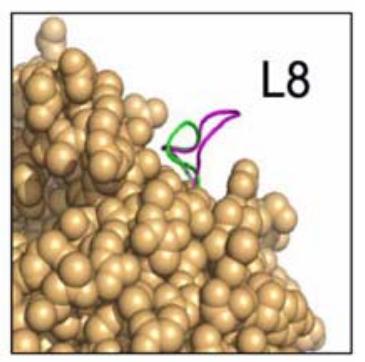

C

(B)

Fig. 5. Comparison between the two 3D-models predicated from motor domains of the cotton GhKCH2 and a fungal kinesin NcKin. (A) Differences between GhKCH2 and NcKin motor domains. The whole molecular structures are in the central, and L8 and Switch II cluster (L11- $\alpha 4-\mathrm{L} 12-\alpha 5)$ are indicated (green for NcKin, purple for GhKCH2). The local amplified images of L12 and L8 are on the left and right, respectively. (B) Sequence alignment of L8 and Switch II in NcKin and GhKCH2 motor domains. The identical amino acid residues for both sequences are highlighted with black background.

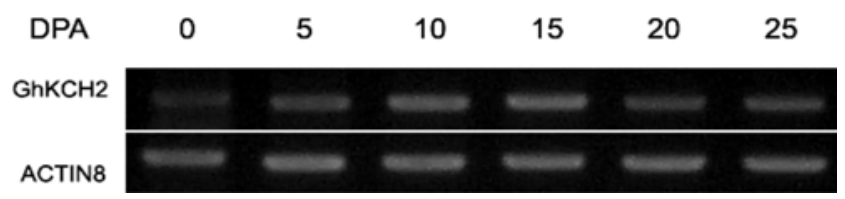

Fig. 6. Expression levels of $\mathrm{GhKCH} 2$ gene at different stages of fiber development. Total RNA was extracted from $0,5,10,15$, 20, 25 DPA fibers with an RNeasy plant mini kit, and then RTPCR was carried out using the GhKCH2 specific primers. The actin8 cDNA was used to normalize the amount of templates added in PCR reactions.

provides spatial information to the cellulose-synthesizing machinery with the plasma membrane of elongating cells (Fisher and Cyr, 1998). In 2002, a kinesin-like protein FRA1 from Arabidopsis was reported to be essential for oriented deposition of cellulose microfibrils and cell wall strength (Zhong et al., 2002). As a plant-specific microtubule motor, GhKCH2 might participate in microtubule dynamics and organization, or transport materials during cellulose deposition and cell wall formation.

Acknowledgments We would like to thank Dr. Ziding Zhang (China Agriculture University, Beijing) and his lab members for the excellent assistance in the molecular model analysis. We are most grateful to Dr. David P. Crockett
(Department of Neuroscience and Cell Biology, UMDNJ-RW Johnson Medical School) for editorial corrections. This research was supported by grants from the Ministry of Science and Technology of China (Project No. JY03-A-03) and the National Natural Science Foundation of China (Project No. 30370708, 30671049, 30421002).

\section{References}

Bloom, G. S. and Endow, S. A. (1994) Motor proteins. 1: kinesins. Protein Profile 1, 1059-1116.

Case, R. B., Rice, S., Hart, C. L., Ly, B. and Vale, R. D. (2000) Role of the kinesin neck linker and catalytic core in microtubule-based motility. Curr. Biol. 10, 157-160.

Castoldi, M. and Popov, A. V. (2003) Purification of brain tubulin through two cycles of polymerization-depolymerization in a high-molarity buffer. Protein Expr. Purif. 32, 83-88.

Emons, A. M. and Mulder, B. M. (2000) How the deposition of cellulose microfibrils builds cell wall architecture. Trends Plant Sci. 5, 35-40.

Endow, S. A. (1999) Determinants of molecular motor directionality. Nat. Cell Biol. 1, E163-167.

Fisher, D. D. and Cyr, R. J. (1998) Extending the microtubule/ microfibril paradigm cellulose synthesis is required for normal cortical microtubule alignment in elongating cells. Plant Physiol. 116, 1043-1051.

Gertel, E. T. and Green, P. B. (1977) Cell growth pattern and wall microfibrillar arrangement: experiments with nitella. Plant 
Physiol. 60, 247-254.

Hackney, D. D. (1988) Kinesin ATPase: rate-limiting ADP release. Proc. Natl. Acad. Sci USA 85, 6314-6318.

Kloth, R. H. (1989) Changes in the level of tubulin subunits during development of cotton (Gossypium hirsutum) fiber. Physiol. Plant 76, 37-41.

Klumpp, L. M., Brendza, K. M., Rosenberg, J. M., Hoenger, A. and Gilbert, S. P. (2003). Motor domain mutation traps kinesin as a microtubule rigor complex. Biochemistry 42, 2595-2606.

Kuznetsov, S. A. and Gelfand, V. I. (1986) Bovine brain kinesin is a microtubule-activated ATPase. Proc. Natl. Acad. Sci. USA 83, 8530-8534.

Lawrence, C. J., Dawe, R. K., Christie, K. R., Cleveland, D. W., Dawson, S. C., Endow, S. A., Goldstein, L. S., Goodson, H. V., Hirokawa, N., Howard, J., Malmberg, R. L., McIntosh, J. R., Miki, H., Mitchison, T. J., Okada, Y., Reddy, A. S., Saxton, W. M., Schliwa, M., Scholey, J. M., Vale, R. D., Walczak, C. E. and Wordeman, L. (2004) A standardized kinesin nomenclature. J. Cell Biol. 167, 19-22.

LeBel, D., Poirier, G. G. and Beaudoin, A. R. (1978) A convenient method for the ATPase assay. Anal. Biochem. 85, 86-89.

Ma, Y. Z. and Taylor, E. W. (1995) Mechanism of microtubule kinesin ATPase. Biochemistry 34, 13242-13251.

Marx, A., Muller, J. and Mandelkow, E. (2005). The structure of microtubule motor proteins. Adv. Protein Chem. 71, 299-344.

Matsui, K., Collings, D. and Asada, T. (2001). Identification of a novel plant-specific kinesin-like protein that is highly expressed in interphase tobacco BY-2 cells. Protoplasma 215, 105-115.

Matthies, H. J. G., Baskin, R. J. and Hawley, R. S. (2001) Orphan kinesin NOD lacks motile properties but does possess a microtubule-stimulated ATPase activity. Mol. Biol. Cell 12, 4000-4012.

Mitsui, H., Nakatani, K., Yamaguchi-Shinoza, K., Shinozaki, K., Nishikawa, K. and Takahashi, H. (1994) Sequencing and characterization of the kinesin-related genes katB and katC of Arabidopsis thaliana. Plant Mol. Biol. 25, 865-876.

Mitsui, H., Yamaguchi-Shinoza, K., Shinozaki, K., Nishikawa, K. and Takahashi, H. (1993) Identification of a gene family (kat) encoding kinesin-like proteins in Arabidopsis thaliana and the characterization of secondary structure of KatA. Mol. Gen. Genet. 238, 362-368.

Ni, C. Z., Wang, H. Q., Xu, T., Qu, Z. and Liu, G. Q. (2005) AtKP1, a kinesin-like protein, mainly localizes to mitochondria in Arabidopsis thaliana. Cell Res. 15, 725-733.

Pan, R., Lee, Y. R. and Liu, B. (2004) Localization of two homologous Arabidopsis kinesin-related proteins in the phragmoplast. Planta 220, 156-164.

Preuss, M. L., Kovar, D. R., Lee, Y. R., Staiger, C. J., Delmer, D. P. and Liu, B. (2004) A plant-specific kinesin binds to actin microfilaments and interacts with cortical microtubules in cotton fibers. Plant Physiol. 136, 3945-3955.

Reddy, A. S. and Day, I. S. (2001). Kinesins in the Arabidopsis genome: a comparative analysis among eukaryotes. $B M C$
Genomics 2, 2.

Reddy, A. S. N. and Day, I. S. (2000) The role of the cytoskeleton and a molecular motor in trichome morphogenesis. Trends Plant Sci. 5, 503-505.

Richardson, D. N., Simmons, M. P. and Reddy, A. S. (2006) Comprehensive comparative analysis of kinesins in photosynthetic eukaryotes. BMC Genomics 7, 18.

Sack, S., Kull, F. J. and Mandelkow, E. (1999) Motor proteins of the kinesin family. Structures, variations, and nucleotide binding sites. Eur. J. Biochem. 262, 1-11.

Sack, S., Muller, J., Marx, A., Thormahlen, M., Mandelkow, E. M., Brady, S. T. and Mandelkow, E. (1997) X-ray structure of motor and neck domains from rat brain kinesin. Biochemistry 36, 16155-16165.

Seagull, R. W. (1992) A quantitative electron microscopic study of changes in microtubule arrays and wall microfibril orientation during in vitro cotton Fiber development. J. Cell Sci 101, 561577.

Shimizu, T., Sablin, E., Vale, R. D., Fletterick, R., Pechatnikova, E. and Taylor, E. W. (1995) Expression, purification, ATPase properties, and microtubule-binding properties of the ncd motor domain. Biochemistry 34, 13259-13266.

Song, Y. H., Marx, A., Muller, J., Woehlke, G., Schliwa, M., Krebs, A., Hoenger, A. and Mandelkow, E. (2001) Structure of a fast kinesin: implications for ATPase mechanism and interactions with microtubules. EMBO J. 20, 6213-6225.

Tamura, K., Nakatani, K., Mitsui, H., Ohashi, Y. and Takahashi, H. (1999). Characterization of katD, a kinesin-like protein gene specifically expressed in floral tissues of Arabidopsis thaliana. Gene 230, 23-32.

Umeki, N., Mitsui, T., Umezu, N., Kondo, K. and Maruta, S. (2006) Preparation and characterization of a novel rice plantspecific kinesin. J. Biochem. (Tokyo) 139, 645-654.

Vale, R.D., and Fletterick, R.J. (1997) The design plan of kinesin motors. Annu. Rev. Cell Dev. Biol. 13, 745-777.

Vos, J. W., Safadi, F., Reddy, A. S. and Hepler, P. K. (2000) The kinesin-like calmodulin binding protein is differentially involved in cell division. Plant Cell 12, 979-990.

Walker, R. A., Salmon, E. D. and Endow, S. A. (1990) The Drosophila claret segregation protein is a minus-end directed motor molecule. Nature 347, 780-782.

Williams, R. C. J. and Lee, J. C. (1982) Preparation of tubulin from brain; in Methods in Enzymology, Giovanni, D. S., Sidney, P. C., Nathan, O. K. (eds.), pp. 376-385, Academic Press, Inc., New York, USA

Yang, J. T., Laymon, R. A. and Goldstein, L. S. (1989) A threedomain structure of kinesin heavy chain revealed by DNA sequence and microtubule binding analyses. Cell 56, 879-889.

Zhong, R., Burk, D. H., Morrison, W. H., 3rd, and Ye, Z. H. (2002) A kinesin-like protein is essential for oriented deposition of cellulose microfibrils and cell wall strength. Plant Cell 14, 3101-3117. 